\title{
On the existence of periodic and homoclinic orbits for first order superquadratic Hamiltonian systems
}

\author{
Adel Daouas
}

\begin{abstract}
In this paper we consider the following Hamiltonian system

$$
J \dot{u}+B(t) u+\nabla W(t, u)=0 .
$$

Under a new superquadratic assumption on the potential, we prove that (HS) has a sequence of subharmonics. This will be done using a minimax result in critical point theory. Also, we study the asymptotic behavior of these subharmonics and we establish the existence of a homoclinic orbit for (HS). Previous results in the topic, mainly those due to Rabinowitz and Tanaka, are significantly improved.
\end{abstract}

Mathematical Subject Classification. 35B38, 34C37, 70H05.

Keywords. Hamiltonian system, Homoclinic orbit, Subharmonics, Critical point.

\section{Introduction and main results}

This paper deals with the existence of subharmonic solutions (i.e. $k T$-periodic solutions, $k \in \mathbb{N}$ ) and their asymptotic behavior for the following first order Hamiltonian system

$$
J \dot{u}+B(t) u+\nabla W(t, u)=0,
$$

where $W \in C^{1}\left(\mathbb{R} \times \mathbb{R}^{2 N}, \mathbb{R}\right)$ is $T$-periodic in the $t$-variable, $B$ is a continuous $T$-periodic and symmetric $2 N \times 2 N$-matrix function, and $J$ is the standard symplectic matrix

$$
J=\left(\begin{array}{cc}
0 & -I_{N} \\
I_{N} & 0
\end{array}\right) .
$$

Recall that a solution $u$ of $(H S)$ is said to be homoclinic to 0 if $u \not \equiv 0$ and $u(t) \longrightarrow 0$ as $|t| \longrightarrow \infty$. 
The problem of finding subharmonic and homoclinic solutions for $(H S)$ has been the object of many works under different assumptions on the growth of $W$ at infinity (see $[4-9,12,14,15,17]$ and references therein). Most of them treat the superquadratic case using the so-called Ambrosetti-Rabinowitz condition, that is, there exists $\mu>2$ such that

$$
0<\mu W(t, x) \leq(\nabla W(t, x), x), \quad \text { for all } \quad t \in \mathbb{R} \text { and } x \in \mathbb{R}^{2 N} \backslash\{0\} .
$$

Here, the superquadraticity condition $(A R)$ will be replaced by a new weaker one firstly introduced in [1] to establish the existence of a sequence of subharmonics for $(H S)$. Also, the existence of a homoclinic orbit for $(H S)$ is proved when the matrix $B$ satisfies

$$
B \text { is independent of } t \text { and } \sigma(J B) \cap i \mathbb{R}=\emptyset,
$$

where $\sigma(B)$ denotes the spectrum of $B$. This work is motivated by the results of $[12,15]$ mainly.

Definition 1.1. A vector field $V$ defined on $\mathbb{R}^{2 N}$ is called positive if $(V x, x)>0$, for all $x \in \mathbb{R}^{2 N} \backslash\{0\}$. We call $V$ is a normalized positive vector field if $V$ is positive, linear and satisfies the following conditions:

$\left(V_{1}\right) J V=V J$

$\left(V_{2}\right)(V x, x)=(x, x), \quad \forall x \in \mathbb{R}^{2 N}$.

Throughout this paper $(\cdot, \cdot)$ denotes the standard inner product in $\mathbb{R}^{2 N}$ and $|\cdot|$ is the induced norm.

Definition 1.2. Let $V$ a normalized positive vector field on $\mathbb{R}^{2 N}$ and $\left\{\phi_{s}\right\}$ its flow, $\mu>0$ be a constant and $h \in C\left(\mathbb{R}^{2 N}, \mathbb{R}\right)$. We call $h$ a positive homogenous function of degree $\mu$ with respect to $V$ if $h(x)>0$ for all $x \in \mathbb{R}^{2 N} \backslash\{0\}$ and

$$
h\left(\phi_{s} x\right)=e^{s \mu} h(x) \quad \forall x \in \mathbb{R}^{2 N}, \forall s \in \mathbb{R} .
$$

If $V x=x$ then $\phi_{s} x=e^{s} x$ and we obtain the classical definition of homogenous function of degree $\mu$.

Theorem 1.3. We assume that $W$ satisfies the following assumptions:

There exist a normalized positive vector field $V$, constants $\left(H_{1}\right) \quad \mu>2$ and $R>1$ such that

$$
0<\mu W(t, x) \leq(\nabla W(t, x), V x), \quad \forall|x| \geq R,
$$

$\left(H_{2}\right) W(t, x)=o\left(|x|^{2}\right)$ as $x \longrightarrow 0$ uniformly in $t$

$\left(H_{3}\right) W(t, x) \geq 0, \forall(t, x) \in \mathbb{R} \times \mathbb{R}^{2 N}$,

there exists

$\left(H_{4}\right) c>0$ such that

$$
|\nabla W(t, x)| \leq c(\nabla W(t, x), V x), \quad \forall|x| \geq R .
$$

Then there is a sequence $\left(k_{j}\right) \subset \mathbb{N}, k_{j} \longrightarrow \infty$, and corresponding distinct $k_{j} T$ periodic solutions of the system $(H S)$. 
The assumption $\left(\mathrm{H}_{4}\right)$ is new, as far as the author is aware, and it can be replaced by the following one firstly introduced by Xu in [16].

$\left(H_{5}\right) \quad \liminf _{|x| \rightarrow \infty} \frac{W_{t}(t, x)}{|x|^{\mu} W(t, x)}=0$, or $\quad \limsup _{|x| \rightarrow \infty} \frac{W_{t}(t, x)}{|x|^{\mu} W(t, x)}=0$.

Precisely, assume that $B$ is of class $C^{1}$, we have

Theorem 1.4. Under the assumptions $\left(H_{1}\right)-\left(H_{3}\right)$ and $\left(H_{5}\right)$, the conclusion of Theorem 1.3 holds.

In the case where $W$ is independent of $t$, the assumption $\left(H_{5}\right)$ is naturally satisfied, so, we obtain

Theorem 1.5. Suppose $W$ is independent of $t$ and satisfies $\left(H_{1}\right)-\left(H_{3}\right)$, then the conclusion of Theorem 1.3 holds.

Corollary 1.6. [2, Theorem 1.3]. Suppose $W$ is independent of t, satisfies $\left(H_{1}\right)-$ $\left(H_{3}\right)$ and $B=0$, then the system $(H S)$ possesses a nonconstant T-periodic solution.

In recent years many authors studied the existence of homoclinic orbits for Hamiltonian systems. Most of them treat the second order systems. The first order systems $(H S)$ is studied by Coti-Zelati et al. in [5] under an assumption of strict convexity on $W(t, x)$ with respect to $x$ and using a dual variational formulation. Also, the problem was considered by Hofer and Wysocki in [9], where they made the following assumptions essentially

$\left(H_{3}^{\prime}\right)$ there exist $\alpha \geq \mu, k_{1}>0$ such that

$$
W(t, x) \geq k_{1}|x|^{\alpha}, \quad \forall(t, x) \in \mathbb{R} \times \mathbb{R}^{2 N},
$$

$\left(H_{4}^{\prime}\right)$ there exists $c>0$ such that

$$
|\nabla W(t, x)| \leq c|x|^{\mu-1}, \quad \forall(t, x) \in \mathbb{R} \times \mathbb{R}^{2 N} .
$$

In their approach, they used first order elliptic system and nonlinear Fredholm operator theory. Later, Tanaka in [15], kept the assumption $\left(H_{3}^{\prime}\right)$ and changed $\left(H_{4}^{\prime}\right)$ by a weaker condition similar to $\left(H_{4}\right)$. He used a minimax result in critical point theory to establish the existence of a sequence of subharmonics which converges to a nontrivial homoclinic orbit. In all above works, the assumption $(A R)$ is fundamental. In this paper, we use a globally version of the assumption $\left(H_{1}\right)$ which is weaker than $(A R)$, (see Remark 1.9), we drop the restrictive assumption $\left(H_{3}^{\prime}\right)$ and we assume that all the eigenvalues of the constant matrix $J B$ are not purely imaginary. Furthermore, the following technical condition is needed:

$\left(H_{6}\right)$ There exists a normalized positive vector field $V$ such that

$$
(B x, V x) \geq(B x, x), \quad \forall x \in \mathbb{R}^{2 N} .
$$

The existence of a nontrivial homoclinic solution for $(H S)$ will be proved as it was done in [15]. Precisely we have: 
Theorem 1.7. Suppose $B$ satisfies (1.1) and $\left(H_{6}\right)$ and $W$ satisfies

$\left(H_{1}^{\prime}\right)$ there exists a constant $\mu>2$ such that

$$
0<\mu W(t, x) \leq(\nabla W(t, x), V x), \quad \forall x \in \mathbb{R}^{2 N} \backslash\{0\},
$$

$\left(H_{2}^{\prime}\right) \nabla W(t, x)=o(|x|)$ as $x \longrightarrow 0$ uniformly in $t$, and $\left(H_{4}\right)$ or $\left(H_{5}\right)$. Then the system $(H S)$ possesses a nontrivial homoclinic solution emanating from 0 .

An interesting class of Hamiltonians satisfying the assumption $\left(H_{1}\right)$ are the homogenous ones in the sense of Definition 1.2. This class was studied in [3] in the autonomous case, where a result of existence of periodic solution for (HS) was proved [3, Theorem 1.5]. The following theorem completes and improves the result in [3].

Theorem 1.8. Suppose $W$ is a positive homogenous function of degree $\mu>2$ with respect to a normalized positive vector field $V$, then there exist a sequence $\left(k_{j}\right) \subset \mathbb{N}, k_{j} \longrightarrow \infty$, and corresponding distinct $k_{j} T$ periodic solutions of the system (HS). Moreover, if $B$ satisfies the assumptions (1.1) and $\left(H_{6}\right)$, then the system $(H S)$ possesses a nontrivial homoclinic solution emanating from 0.

Remark 1.9. It is obvious that if $V x=x$, then $\left(H_{1}\right)$ becomes the classical condition $(A R)$ and $\left(H_{6}\right)$ is naturally satisfied. Example 1.4 in [2] shows that $\left(H_{1}\right)$ is weaker than $(A R)$ essentially. Moreover, let $N=1$,

$$
V=\left(\begin{array}{ll}
1 & 1 \\
-1 & 1
\end{array}\right) \quad \text { and } \quad B=\left(\begin{array}{ll}
0 & 1 \\
-1 & 0
\end{array}\right) \text {. }
$$

A straightforward computation shows that $V$ and $B$ satisfy the assumptions (1.1) and $\left(H_{6}\right)$. Therefore the above theorems improve the results in $[12,15,17]$ mainly.

Remark 1.10. In [15], it is shown that condition (1.1) implies that, in an appropriate function space, the operator $-J \frac{d}{d t}-B$ is invertible, i.e., $\sigma\left(-J \frac{d}{d t}-B\right) \cap$ $(-\alpha, \alpha)=\emptyset$ for some $\alpha>0$. In a recent work, Ding and Willem [7] relaxed the above condition. They allowed the matrix $B$ to be $t$-dependent, periodic and such that $\sigma\left(-J \frac{d}{d t}-B\right) \cap(0, \alpha)=\emptyset$ for some $\alpha>0$ and they established the existence of a homoclinic orbit for $(H S)$. We note that our approach can be modified so that it includes systems with the more general linear term like in [8]. As it is mentioned in [17], this needs to change our functional setting. So, in order to minimize technicalities, we restrict our attention to the case of systems with invertible linear part.

\section{Functional framework and variational formulation}

Let $S_{T}=\mathbb{R} /(T \mathbb{Z})$ and $E_{T}=H^{1 / 2}\left(S_{T}, \mathbb{R}^{2 N}\right)$ be the Sobolev space of all $T$-periodic $\mathbb{R}^{2 N}$-valued functions $u$ in $L^{2}\left(S_{T}, \mathbb{R}^{2 N}\right)$ whose Fourier series

$$
u(t)=\sum_{j=-\infty}^{j=+\infty} \exp \left(\frac{2 j \pi t J}{T}\right) a_{j}, \quad a_{j} \in \mathbb{R}^{2 N}
$$


satisfies

$$
\|u\|_{E_{T}}^{2}:=T\left|a_{0}\right|^{2}+T \sum_{j=-\infty}^{j=+\infty}|j| \cdot\left|a_{j}\right|^{2}<+\infty .
$$

The inner product on $E_{T}$ is defined by

$$
(u, v)_{E_{T}}=T\left(a_{0}, b_{0}\right)+T \sum_{j=-\infty}^{j=+\infty}|j|\left(a_{j}, b_{j}\right)
$$

where

$$
v(t)=\sum_{j=-\infty}^{j=+\infty} \exp \left(\frac{2 j \pi t J}{T}\right) b_{j}, \quad b_{j} \in \mathbb{R}^{2 N} .
$$

It is well known that $E_{T}$ is compactly embedded in $L^{\gamma}\left(S_{T}, \mathbb{R}^{2 N}\right)$ for every $\gamma \in[1,+\infty)$ and there exists a constant $c_{\gamma}$ such that

$$
\|u\|_{L^{\gamma}} \leq c_{\gamma}\|u\|_{E_{T}}, \quad \forall u \in E_{T}
$$

Define two self-adjoint operators $\mathcal{A}, \mathcal{B} \in L\left(E_{T}\right)$ by extending the bilinear forms

$$
(\mathcal{A} u, v)=\int_{0}^{T}(-J \dot{u}, v) d t, \quad(\mathcal{B} u, v)=\int_{0}^{T}(B(t) u, v) d t, \quad \forall u, v \in E_{T} .
$$

By [10] and the standard spectral theory, $\mathcal{B}$ is compact on $E_{T}$. Denote the eigenvalues of $\mathcal{A}-\mathcal{B}$ on $E$ by

$$
\cdots \leq \lambda_{-2} \leq \lambda_{-1}<0=\left(\lambda_{0}\right)<\lambda_{1} \leq \lambda_{2} \cdots
$$

where when $\operatorname{dim} \operatorname{ker}(\mathcal{A}-\mathcal{B})=0, \lambda_{0} \notin \sigma(\mathcal{A}-\mathcal{B})$. Let $\left\{e_{ \pm j}\right\}_{j \in \mathbb{N}}$ be the eigenvectors of $\mathcal{A}-\mathcal{B}$ corresponding to $\left\{\lambda_{ \pm j}\right\}$, respectively. Define $E^{+}=$span $\left\{e_{1}, e_{2}, \ldots\right\}, E^{-}=\operatorname{span}\left\{e_{-1}, e_{-2}, \ldots\right\}, E^{0}=\operatorname{ker}(\mathcal{A}-\mathcal{B})$. Hence there exist an orthogonal decomposition $E_{T}=E^{+} \oplus E^{-} \oplus E^{0}$ with $\operatorname{dim} E^{0}<\infty$, dim $E^{+}=\operatorname{dim} E^{-}=\infty$ and an equivalent inner product in $E_{T}$, denoted by $(\cdot, \cdot)$ and defined by

$$
(u, v)=\left((\mathcal{A}-\mathcal{B}) u^{+}, v^{+}\right)_{E_{T}}-\left((\mathcal{A}-\mathcal{B}) u^{-}, v^{-}\right)_{E_{T}}+\left(u^{0}, v^{0}\right)_{E_{T}} .
$$

for all $u=u^{+}+u^{-}+u^{0}, v=v^{+}+v^{-}+v^{0}$ in $E^{+} \oplus E^{-} \oplus E^{0}$. Hence, we have

$$
\int_{0}^{T}(-J \dot{u}-B(t) u, u) d t=((\mathcal{A}-\mathcal{B}) u, u)_{E_{T}}=\left\|u^{+}\right\|^{2}-\left\|u^{-}\right\|^{2}
$$

where $\|\cdot\|$ is the norm induced by $(\cdot, \cdot)$. Let $V$ be the normalized vector field in $\left(H_{1}\right)$, then $V$ is an invertible endomorphism on $\mathbb{R}^{2 N}$ and there exist constants $a, b>0$ such that

$$
a|x| \leq|V x| \leq b|x|, \quad \forall x \in \mathbb{R}^{2 N} .
$$

Define a vector field $\widetilde{V}$ on $E_{T}$ by $(\widetilde{V} u)(t)=(V u)(t)$. Using conditions $\left(V_{1}\right),\left(V_{2}\right)$ and the Fourier series, a direct computation gives 
Lemma 2.1. [1] For all $u \in E_{T}$, we have

i) $\quad(\mathcal{A} u, \widetilde{V} u)_{E_{T}}=(\mathcal{A} u, u)_{E_{T}}$,

ii) $\quad a\|u\|_{E_{T}} \leq\|\widetilde{V} u\|_{E_{T}} \leq b\|u\|_{E_{T}}$.

Since the growth rate of the the function $W$ at $\infty$ is not restricted, we introduce modification of $W$ as follows. Let $K \geq 1$ and $\chi_{K} \in C^{\infty}(\mathbb{R}, \mathbb{R})$ such that $\chi_{K}(s)=1$ for $s \leq K, \chi_{K}(s)=0$ for $s \geq K+1$, and $\chi_{K}^{\prime}(s) \leq 0$. Define

$$
W_{K}(t, x)=\chi_{K}(|x|) W(t, x)+\left(1-\chi_{K}(|x|)\right) r_{K}|x|^{\mu},
$$

where

$$
r_{K}=\max _{K \leq|x| \leq K+1} \frac{W(t, x)}{|x|^{\mu}} .
$$

Lemma 2.2. $W_{K}$ satisfies all assumption satisfied by $W$. Furthermore, by $\left(H_{1}\right)$, there are constants $a_{1}, a_{2}>0$ independent of $K$ such that

$$
W_{K}(t, x) \geq a_{1}|x|^{\mu}-a_{2}, \quad \forall(t, x) \in \mathbb{R} \times \mathbb{R}^{2 N} .
$$

Proof. The properties $\left(H_{2}\right),\left(H_{2}^{\prime}\right),\left(H_{3}\right)$ and $\left(H_{5}\right)$ are easy to prove. For the proof of $\left(H_{1}\right)$ and $(2.3)$ see [2]. It remains only to prove the new condition $\left(H_{4}\right)$. Indeed, by the definition of $\chi_{K}$ and $r_{K}$ we have $\chi_{K}^{\prime}(|x|)\left(W(t, x)-r_{K}|x|^{\mu}\right) \geq 0$ and therefore

$$
\begin{aligned}
\left|\nabla W_{K}(t, x)\right| \leq & \chi_{K}^{\prime}(|x|)\left(W(t, x)-r_{K}|x|^{\mu}\right)+\chi_{K}(|x|)|\nabla W(t, x)| \\
& +\mu\left(1-\chi_{K}(|x|)\right) r_{K}|x|^{\mu-1} .
\end{aligned}
$$

On the other hand, for $|x| \geq R$,

$$
\begin{aligned}
\left(\nabla W_{K}(t, x), V x\right) \geq & \chi_{K}^{\prime}(|x|)\left(W(t, x)-r_{K}|x|^{\mu}\right)+\chi_{K}(|x|)(\nabla W(t, x), V x) \\
& +\mu\left(1-\chi_{K}(|x|)\right) r_{K}|x|^{\mu-1} .
\end{aligned}
$$

So, by $\left(H_{4}\right)$, we obtain

$$
\left|\nabla W_{K}(t, x)\right| \leq \widetilde{c}\left(\nabla W_{K}(t, x), V x\right),
$$

where $\widetilde{c}=c+1$.

Now, to prove Theorem 1.3, it suffices to find a nontrivial solution for

$$
J \dot{u}+B(t) u+\nabla W_{K}(t, u)=0,
$$

with $\|u\|_{L^{\infty}} \leq K$. Define the functional $I_{K}: E_{T} \longrightarrow \mathbb{R}$, by

$$
\begin{aligned}
I_{K}(u) & =\frac{1}{2} \int_{0}^{T}(-J \dot{u}-B(t) u, u) d t-\int_{0}^{T} W_{K}(t, u) d t \\
& =\frac{1}{2}\left(\left\|u^{+}\right\|^{2}-\left\|u^{-}\right\|^{2}\right)-\int_{0}^{T} W_{K}(t, u) d t .
\end{aligned}
$$

By the form of $W_{K}$ at infinity, we know that $I_{K} \in C^{1}\left(E_{T}, \mathbb{R}\right)$, and the critical points of the functional $I_{K}$ are the $T$-periodic solutions of $(H S)_{K}$ (see $\left.[11,13]\right)$. 
Moreover,

$$
\begin{aligned}
I_{K}^{\prime}(u)(v) & =\int_{0}^{T}(-J \dot{u}-B(t) u, v) d t-\int_{0}^{T}\left(\nabla W_{K}(t, u), v\right) d t \\
& =\left(u^{+}-u^{-}, v\right)-\int_{0}^{T}\left(\nabla W_{K}(t, u), v\right) d t, \quad \forall u, v \in E_{T} .
\end{aligned}
$$

Using the ideas of [16], we have the following two Lemmas on the $C^{0}$ bound of the periodic solutions of $(H S)_{K}$.

Lemma 2.3. Suppose $W$ satisfies $\left(H_{1}\right)$ and $\left(H_{4}\right)$. If $u_{K}$ is a T-periodic solution of $(H S)_{K}$ such that

$$
\int_{0}^{T} W_{K}\left(t, u_{K}\right) d t \leq M, \int_{0}^{T}\left(\nabla W_{K}\left(t, u_{K}\right), \widetilde{V} u_{k}\right) d t \leq M
$$

then there is a constant $L$ independent of $K$ and dependent on $M$ only such that

$$
\left\|u_{K}\right\|_{L^{\infty}} \leq L
$$

Proof. Let $u_{K}$ a $T$-periodic solution of $(H S)_{K}$ satisfying (2.5). By (2.3), we have

$$
M \geq \int_{0}^{T} W_{K}\left(t, u_{K}\right) d t \geq a_{1} \int_{0}^{T}\left|u_{K}(t)\right|^{\mu} d t-a_{2} T \geq a_{1} T\left(\min _{t \in S_{T}}\left|u_{K}(t)\right|\right)^{\mu}-a_{2} T,
$$

which implies

$$
\min _{t \in S_{T}}\left|u_{K}(t)\right| \leq C_{0},
$$

where $C_{0}$ is independent of $K$. We claim that there exists a constant $L$ independent of $K$ and depends on $M$ only verifying $\left\|u_{K}\right\|_{L^{\infty}} \leq L$. If not, without loss of generality, using (2.6), we may assume that there are $K_{n}, L_{n} \in \mathbb{R}, t_{n} \in S_{T}$ such that

$$
\left\{\begin{array}{l}
L_{n} \longrightarrow+\infty \text { as } n \longrightarrow+\infty \\
\left|u_{n}(t)\right| \geq R, \forall t \in\left[0, t_{n}\right) \\
\left|u_{n}(0)\right|=R,\left|u_{n}\left(t_{n}\right)\right|=L_{n}
\end{array}\right.
$$

where $u_{n}:=u_{K_{n}}$.

Now, since $u_{n}$ is a solution of $(H S)_{K_{n}}$ we have

$$
\begin{aligned}
\left|u_{n}\left(t_{n}\right)\right|-\left|u_{n}(0)\right| & =\int_{0}^{t_{n}} \frac{d}{d s}\left|u_{n}(s)\right| d s \\
& =\int_{0}^{t_{n}} \frac{\left(u_{n}(s), \dot{u}_{n}(s)\right)}{\left|u_{n}(s)\right|} d s \\
& \leq \int_{0}^{t_{n}}\left|\dot{u}_{n}(s)\right| d s \\
& \leq \int_{0}^{t_{n}}\left(\left|B(s) u_{n}(s)\right|+\left|\nabla W_{K_{n}}\left(s, u_{n}(s)\right)\right|\right) d s .
\end{aligned}
$$


and by $\left(H_{4}\right)$ we get

$$
L_{n} \leq\|B\|_{L^{\infty}} \int_{0}^{t_{n}}\left|u_{n}(s)\right|^{\mu} d s+c \int_{0}^{t_{n}}\left(\nabla W_{K_{n}}\left(s, u_{n}(s)\right), \tilde{V}\left(u_{n}(s)\right)\right) d s+R .
$$

From the proof of Lemma 2.2, we know that

$$
W_{K}(t, x) \geq a_{1}|x|^{\mu}, \forall|x| \geq R .
$$

So, by (2.5), (2.8) and (2.9), we obtain

$$
\begin{aligned}
L_{n} & \leq \frac{\|B\|_{L^{\infty}}}{a_{1}} \int_{0}^{t_{n}} W_{K_{n}}\left(s, u_{n}(s)\right) d s+c \int_{0}^{t_{n}}\left(\nabla W_{K_{n}}\left(s, u_{n}(s)\right), \tilde{V}\left(u_{n}(s)\right)\right) d s+R \\
& \leq \frac{\|B\|_{L^{\infty}}}{a_{1}} M+c M+R .
\end{aligned}
$$

but this contradicts the fact $L_{n} \longrightarrow \infty$ as $n \longrightarrow \infty$.

Lemma 2.4. Suppose $W$ satisfies $\left(H_{1}\right)$ and $\left(H_{5}\right)$. If $u_{K}$ is a T-periodic solution of $(H S)_{K}$ verifying (2.5), then there is a constant $L$ independent of $K$ and dependent on $M$ only such that

$$
\left\|u_{K}\right\|_{L^{\infty}} \leq L
$$

Proof. Define

$$
\sigma(r)=\sup _{|x| \geq r, t \in S_{T}} \frac{W_{t}(t, x)}{|x|^{\mu} W(t, x)}, \quad \delta(r)=\inf _{|x| \geq r, t \in S_{T}} \frac{W_{t}(t, x)}{|x|^{\mu} W(t, x)} .
$$

By $\left(H_{5}\right)$, we have

$$
\lim _{r \longrightarrow+\infty} \sigma(r)=0 \quad \text { or } \quad \lim _{r \longrightarrow+\infty} \delta(r)=0 .
$$

Case I: Suppose that $\lim _{r \longrightarrow+\infty} \sigma(r)=0$. By definition, $\sigma(r)$ decreases to 0. Fix $r_{0}>R$ large enough such that $a_{1}-\sigma\left(r_{0}\right) M>0$. Let $u_{K}$ a $T$-periodic solution of $(H S)_{K}$ verifying (2.5). Note that $u_{K}$ satisfies (2.6). We claim that there exists $L$ independent of $K$ and depend on $M$ only such that $\left\|u_{K}\right\| \leq L$. If not, there exist $K_{n}, L_{n}, a_{n}, b_{n} \in \mathbb{R}$ such that $L_{n} \longrightarrow+\infty$ as $n \longrightarrow+\infty$,

$$
\left(a_{n}, b_{n}\right) \subset\left\{t \in S_{T} ; r_{0}<\left|u_{n}(t)\right|<L_{n}\right\},
$$

$\left|u_{n}\left(a_{n}\right)\right|=r_{0}$ and $\left|u_{n}\left(b_{n}\right)\right|=L_{n}$ for all $n$ large enough, where $u_{n}:=u_{K_{n}}$. Denote

$$
H_{K}(t, x)=\frac{1}{2}(B(t) x, x)+W_{K}(t, x), H(t, x)=\frac{1}{2}(B(t) x, x)+W(t, x) .
$$


In the following, $B_{t}$ and $W_{K, t}$ denote respectively the derivative of $B$ and $W_{K}$ with respect to the $t$-variable. Since $u_{n}$ is a solution of $(H S)_{K_{n}}$, we have

$$
\begin{aligned}
& H_{K_{n}}\left(b_{n}, u_{n}\left(b_{n}\right)\right)-H_{K_{n}}\left(a_{n}, u_{n}\left(a_{n}\right)\right)=\int_{a_{n}}^{b_{n}} \frac{d}{d t} H_{K_{n}}\left(t, u_{n}(t)\right) d t \\
& \quad=\int_{a_{n}}^{b_{n}}\left[\left(\nabla H_{K_{n}}\left(t, u_{n}(t)\right), \dot{u}_{n}(t)\right)+H_{K_{n}, t}\left(t, u_{n}(t)\right] d t\right. \\
& \quad=\int_{a_{n}}^{b_{n}}\left[\frac{1}{2}\left(B_{t}(t) u_{n}(t), u_{n}(t)\right)+W_{K_{n}, t}\left(t, u_{n}(t)\right)\right] d t \\
& \leq \frac{1}{2} \|\left. B_{t}\left|L^{\infty} \int_{a_{n}}^{b_{n}}\right| u_{n}(t)\right|^{2} d t+\int_{a_{n}}^{b_{n}} \sigma\left(\left|u_{n}(t)\right|\right)\left|u_{n}(t)\right|^{\mu} W_{K_{n}}\left(t, u_{n}(t)\right) d t \\
& \quad \leq d \int_{a_{n}}^{b_{n}} W_{K_{n}}\left(t, u_{n}(t)\right) d t+\sigma\left(r_{0}\right) L_{n}^{\mu} \int_{a_{n}}^{b_{n}} W_{K_{n}}\left(t, u_{n}(t)\right) d t \\
& \leq d M+\sigma\left(r_{0}\right) M L_{n}^{\mu},
\end{aligned}
$$

where $d$ is a positive constant. On the other hand, by (2.3), we get

$$
\begin{aligned}
& H_{K_{n}}\left(b_{n}, u_{n}\left(b_{n}\right)\right)-H_{K_{n}}\left(a_{n}, u_{n}\left(a_{n}\right)\right)-\max _{|x| \leq r_{0}, t \in S_{T}}\left|H_{K_{n}}(t, x)\right| \\
& \geq a_{1}\left|u_{n}\left(b_{n}\right)\right|^{\mu}-a_{2}-\frac{1}{2}|| B||_{L^{\infty}}\left|u_{n}\left(b_{n}\right)\right|^{2} \\
& \geq a_{1} L_{n}^{\mu}-a_{2}-\frac{1}{2}|| B \|_{L^{\infty}} L_{n}^{2}-\max _{|x| \leq r_{0}, t \in S_{T}}|H(t, x)| .
\end{aligned}
$$

Combine the inequalities (2.10) and (2.11), we obtain

$$
\left(a_{1}-\sigma\left(r_{0}\right) M\right) L_{n}^{\mu}-\frac{1}{2}\|B\|_{L^{\infty}} L_{n}^{2} \leq a_{2}+c M+\max _{|x| \leq r_{0}, t \in S_{T}}|H(t, x)| .
$$

Since $a_{1}-\sigma\left(r_{0}\right) M>0, \mu>2$ and $L_{n} \longrightarrow \infty$ as $\longrightarrow+\infty$, the last inequality leads to a contradiction and our claim is true.

Case II: Suppose that $\lim _{r \longrightarrow+\infty} \delta(r)=0$. We modify slightly the proof of Case I to obtain the following inequality

$$
\left(a_{1}+\delta\left(r_{0}\right) M\right) L_{n}^{\mu}-\frac{1}{2}\|B\|_{L^{\infty}} L_{n}^{2} \leq a_{2}+c M+\max _{|x| \leq r_{0}, t \in S_{T}}|H(t, x)| .
$$

Using the same argument as in the above, we obtain a contradiction.

In order to prove our main results, we state the following critical point theorem.

Theorem 2.5. [13] Let $E$ be a real Hilbert space with $E=E_{1} \oplus E_{2}$ and $E_{2}=E_{1}^{\perp}$. Suppose $f \in C^{1}(E, \mathbb{R})$, satisfies (PS) condition, and

$\left(f_{1}\right) \quad f(x)=\frac{1}{2}(L x, x)_{E}+\phi(x)$, where $L=L_{1} P_{1}+L_{2} P_{2}$ and $L_{i}: E_{i} \longrightarrow E_{i}$ is bounded and self-adjoint, $i=1,2$,

$\left(f_{2}\right) \phi^{\prime}$ is compact, and

$\left(f_{3}\right)$ thereexistasubsequence $\widetilde{E} \subset E$, sets $S \subset E, Q \subset \widetilde{E}$ and constants $\alpha>\omega$ such that 
(i) $S \subset E_{1}$ and $\left.f\right|_{S} \geq \alpha$,

(ii) $Q$ is bounded and $\left.f\right|_{\partial Q} \leq \omega$,

(iii) $S$ and $\partial Q$ link.

Then

$$
c=\inf _{h \in \Gamma} \sup _{x \in Q} f(h(1, x))
$$

is a critical value of $f$ and $c \geq \alpha$, where $\Gamma$ is defined by $\Gamma=\{h \in C([0,1] \times$ $\left.E, E)|h(0, x)=x, h(1, x)|_{\partial Q}=x, h(t, x)=e^{\theta(t, x) L}+K(t, x)\right\}$.

Here, $\theta \in C([0,1] \times E, E)$ and $K$ is compact.

\section{Proof of main results}

To find a nontrivial critical point of $I_{K}$, we use the above theorem with $E_{1}=$ $E^{+}, E_{2}=E^{-} \oplus E^{0}$. As shown in [13], the functional $I_{K}$ satisfies $\left(f_{1}\right)$ and $\left(f_{2}\right)$.

Lemma 3.1. $I_{K}$ satisfies the $(P S)$ condition, i.e., if $\left\{u_{n}\right\} \subset E$, with $I_{K}^{\prime}\left(u_{n}\right) \longrightarrow$ 0 and $\left|I_{K}\left(u_{n}\right)\right| \leq M$, for some constant $M>0$, then $\left\{u_{n}\right\}$ has a convergent subsequence.

Proof. In this proof $C_{k}, k=1, \ldots, 10$, are positive constants. By the equivalence of norms on $E_{T}$, for $n$ large enough, we have

$$
M+C_{1}\left\|u_{n}\right\| \geq M+\left\|\widetilde{V} u_{n}\right\|_{E} \geq I_{K}\left(u_{n}\right)-\frac{1}{2} I_{K}^{\prime}\left(u_{n}\right)\left(\widetilde{V} u_{n}\right) .
$$

From Lemma 2.1, we receive

$$
\int_{0}^{T}\left(-J \dot{u}_{n}, u_{n}\right) d t=\int_{0}^{T}\left(-J \dot{u}_{n}, \widetilde{V} u_{n}\right) d t .
$$

Then, we obtain

$$
\begin{aligned}
M+C_{1}\left\|u_{n}\right\| \geq & \frac{1}{2} \int_{0}^{T}\left(B(t) u_{n}, \widetilde{V} u_{n}-u_{n}\right) d t-\int_{0}^{T} W_{K}\left(t, u_{n}\right) d t \\
& +\frac{1}{2} \int_{0}^{T}\left(\nabla W_{K}\left(t, u_{n}\right), \widetilde{V} u_{n}\right) d t .
\end{aligned}
$$

By $\left(H_{1}\right)$ and $(2.3)$, we get

$$
\begin{aligned}
M+C_{1}\left\|u_{n}\right\| & \geq \frac{1}{2} \int_{0}^{T}\left(B(t) u_{n}, \widetilde{V} u_{n}-u_{n}\right) d t+\left(\frac{\mu}{2}-1\right) \int_{0}^{T} W_{K}\left(t, u_{n}\right) d t-C_{2} \\
& \geq \frac{1}{2} \int_{0}^{T}\left(B(t) u_{n}, \widetilde{V} u_{n}-u_{n}\right) d t+\left(\frac{\mu}{2}-1\right) a_{1} \int_{0}^{T}\left|u_{n}\right|^{\mu} d t-C_{3} .
\end{aligned}
$$

On the other hand, by (2.2) and Hölder inequality, there is a constant $C_{4}>0$ such that

$$
\left|\int_{0}^{T}\left(B(t) u_{n}, \widetilde{V} u_{n}-u_{n}\right) d t\right| \leq C_{4}\left\|u_{n}\right\|_{L^{\mu}}^{2} .
$$


From (3.1) and (3.2), we obtain

$$
M+C_{1}\left\|u_{n}\right\| \geq-\frac{C_{4}}{2}\left\|u_{n}\right\|_{L^{\mu}}^{2}+\left(\frac{\mu}{2}-1\right) a_{1}\left\|u_{n}\right\|_{L^{\mu}}^{\mu}-C_{3},
$$

which implies

$$
\left\|u_{n}\right\|_{L^{\mu}}^{\mu} \leq C_{5}\left(1+\left\|u_{n}\right\|\right)
$$

Writing $u_{n}=u_{n}^{+}+u_{n}^{-}+u_{n}^{0} \in E^{+} \oplus E^{-} \oplus E^{0}$, since $E^{0}$ is a finite-dimensional space, it follows from (3.3) that

$$
\left\|u_{n}^{0}\right\| \leq C_{6}\left(1+\left\|u_{n}\right\|^{1 / \mu}\right) .
$$

Now, taking $v=u_{n}^{+}$in (2.4), yields

$$
I_{K}^{\prime}\left(u_{n}\right)\left(u_{n}^{+}\right)=\left\|u_{n}^{+}\right\|^{2}-\int_{0}^{T}\left(\nabla W_{K}\left(t, u_{n}\right), u_{n}^{+}\right) d t .
$$

For $n$ large enough, by Hölder inequality and the form of $\nabla W_{K}$ at infinity, we get

$$
\begin{aligned}
\left\|u_{n}^{+}\right\|^{2} & \leq\left\|u_{n}^{+}\right\|+\left(\int_{0}^{T}\left|\nabla W_{K}\left(t, u_{n}\right)\right|^{\frac{\mu}{\mu-1}} d t\right)^{\frac{\mu-1}{\mu}}\left(\int_{0}^{T}\left|u_{n}^{+}\right|^{\mu} d t\right)^{\frac{1}{\mu}} \\
& \leq\left\|u_{n}^{+}\right\|+C_{7}\left(1+\left\|u_{n}\right\|_{L^{\mu}}^{\mu-1}\right)\left\|u_{n}^{+}\right\|_{L^{\mu}} \\
& \leq C_{8}\left(1+\left\|u_{n}\right\|_{L^{\mu}}^{\mu-1}\right)\left\|u_{n}^{+}\right\| .
\end{aligned}
$$

Thus, by (3.3)

$$
\left\|u_{n}^{+}\right\| \leq C_{8}\left(1+\left\|u_{n}\right\|_{L^{\mu}}^{\mu-1}\right) \leq C_{9}\left(1+\left\|u_{n}\right\|^{\frac{\mu-1}{\mu}}\right) .
$$

Analogously, with $v=u_{n}^{-}$in (2.4), yields

$$
\left\|u_{n}^{-}\right\| \leq C_{9}\left(1+\left\|u_{n}\right\|^{\frac{\mu-1}{\mu}}\right) \text {. }
$$

The inequalities (3.4)-(3.6) imply

$$
\left\|u_{n}\right\| \leq\left\|u_{n}^{+}\right\|+\left\|u_{n}^{-}\right\|+\left\|u_{n}^{0}\right\| \leq C_{10}\left(1+\left\|u_{n}\right\|^{\frac{\mu-1}{\mu}}+\left\|u_{n}\right\|^{1 / \mu}\right) .
$$

This shows that the sequence $\left\{u_{n}\right\}$ is bounded in $E_{T}$. Then by a standard argument (see [13]), $\left\{u_{n}\right\}$ has a convergent subsequence.

Lemma 3.2. $I_{K}$ satisfies the condition $\left(f_{3}\right)$.

Proof. By $\left(H_{2}\right)$ and the form of $W_{K}$, for any $\epsilon>0$, there exists $M>0$ such that

$$
W_{K}(t, x) \leq \epsilon|x|^{2}+M|x|^{\mu}, \quad \forall x \in \mathbb{R}^{2 N} .
$$

Using the norm $\|\cdot\|$ instead of $\|\cdot\|_{E},(2.1)$ and (3.7), we get for $u \in E_{1}$,

$$
\begin{aligned}
I_{K}(u) & =\frac{1}{2}\|u\|^{2}-\int_{0}^{T} W_{K}(t, u) d t \geq \frac{1}{2}\|u\|^{2}-\left(\epsilon c_{2}\|u\|^{2}+M c_{\mu}\|u\|^{\mu}\right) \\
& \geq \frac{1}{2}\|u\|^{2}-\left(\epsilon c_{2}+M c_{\mu}\|u\|^{\mu-2}\right)\|u\|^{2} .
\end{aligned}
$$


Choose $\epsilon=\frac{1}{6 c_{2}}, \rho=\left(\frac{1}{6 M c_{\mu}}\right)^{\frac{1}{\mu-2}}$ and denote by $B_{\rho}$ the closed ball in $E_{T}$ of radius $\rho$ centered at origin. Let $S=\partial B_{\rho} \cap E_{1}$, then $I_{K}(u) \geq \alpha=\frac{\rho^{2}}{6}$ for all $u \in S$, and (i) of $\left(f_{3}\right)$ holds.

The proof of (ii) of $\left(f_{3}\right)$ is slightly different from that in [13]. Indeed, to obtain $Q$ with $r_{1}$ and $r_{2}$ independent of $K$, we let $e \in \partial B_{1} \cap E_{1}$ and $u=u^{0}+u^{-} \in E_{2}$, then

$$
I_{K}(u+r e)=\frac{1}{2}\left(r^{2}-\left\|u^{-}\right\|^{2}\right)-\int_{0}^{T} W_{K}(t, u+r e) d t .
$$

By $\left(H_{3}\right)$, it is obvious that $I_{K}(u) \leq 0$ on $E_{2}$. Since $E^{0}$ is finite dimensional, there exists $a_{3}>0$ such that

$$
\|e\| \leq a_{3}\|e\|_{L^{2}}, \quad\left\|u^{0}\right\| \leq a_{3}\left\|u^{0}\right\|_{L^{2}}
$$

for all $u^{0} \in E^{0}$. Moreover, by (2.3), there is $a_{4}>0$ independent of $K$ such that

$$
W_{K}(t, x) \geq a_{3}^{2}|x|^{2}-a_{4}, \forall(t, x) \in \mathbb{R} \times \mathbb{R}^{2 N} .
$$

The orthogonality of $u^{0}, u^{-}$and $e$ in $L^{2}$ and (3.10) imply

$$
\begin{aligned}
\int_{0}^{T} W_{K}(t, u+r e) d t & \geq a_{3}^{2}\|u+r e\|_{L^{2}}^{2}-a_{4} T \\
& \geq a_{3}^{2}\left(\left\|u^{-}\right\|_{L^{2}}^{2}+\left\|u^{0}\right\|_{L^{2}}^{2}+r^{2}\|e\|_{L^{2}}^{2}\right)-a_{4} T .
\end{aligned}
$$

By (3.8), (3.9) and (3.11), we get

$$
\begin{aligned}
I_{K}(u+r e) & \leq \frac{1}{2}\left(r^{2}-\left\|u^{-}\right\|^{2}\right)-\left(\left\|u^{0}\right\|^{2}+r^{2}\right)+a_{4} T \\
& \leq-\frac{1}{2} r^{2}-\frac{1}{2}\|u\|^{2}+a_{4} T
\end{aligned}
$$

for all $r>0$ and $u \in E_{2}$. Let $r_{1}=r_{2}=\sqrt{2 a_{4} T}$, then $I_{K}(u+r e) \leq 0$ either $r \geq r_{1}$ or $\|u\| \geq r_{2}$. Consequently $I_{K} \leq 0 \equiv \omega$ on $\partial Q$, where $Q=\{$ re $; r \in$ $\left.\left[0, r_{1}\right]\right\} \oplus\left(B_{r_{2}} \cap E_{2}\right)$. By Lemma 6.27 of [13], $S$ and $\partial Q$ link and (ii) and (iii) of $\left(f_{3}\right)$ hold.

Proof of Theorem 1.3. The functional $I_{K}$ satisfies the assumptions of Theorem 2.5, so, it possesses a critical value $c_{K}>0$ and corresponding nontrivial critical point $u_{K}$. It remains to prove that $u_{K}$ satisfies $(H S)$ for appropriately chosen $K$. Since $h(t, x) \equiv x$ belongs to $\Gamma$, one has

$$
\begin{aligned}
c_{K}=I_{K}\left(u_{K}\right) & \leq \sup _{x \in Q} I_{K}(x) \\
& \leq \sup _{0 \leq r \leq r_{1},\left\|u^{-}+u^{0}\right\| \leq r_{2}} \frac{1}{2}\left(r^{2}-\left\|u^{-}\right\|^{2}\right)-\int_{0}^{T} W_{K}\left(t, u^{0}+u^{-}+r e\right) d t \\
& \leq \frac{1}{2} r_{1}^{2} .
\end{aligned}
$$


Note that $r_{1}$ and $r_{2}$ are independent of $K$. Arguing as in (3.1)- (3.2), we get

$$
\begin{aligned}
\frac{1}{2} r_{1}^{2} & \geq c_{K}=I_{K}\left(u_{K}\right)-\frac{1}{2} I_{K}^{\prime}\left(u_{K}\right)\left(\widetilde{V} u_{k}\right) \\
& \geq-\frac{C_{4}}{2}\left\|u_{K}\right\|_{L^{\mu}}^{2}+\left(\frac{1}{2}-\frac{1}{\mu}\right) \int_{0}^{T}\left(\nabla W_{K}\left(t, u_{K}\right), \widetilde{V} u_{k}\right) d t-C_{2} \\
& \geq-\frac{C_{4}}{2}\left\|u_{K}\right\|_{L^{\mu}}^{2}+\left(\frac{\mu}{2}-1\right) \int_{0}^{T} W_{K}\left(t, u_{K}\right) d t-C_{2} \\
& \geq-\frac{C_{4}}{2}\left\|u_{K}\right\|_{L^{\mu}}^{2}+\left(\frac{\mu}{2}-1\right) a_{1}\left\|u_{K}\right\|_{L^{\mu}}^{\mu}-C_{3} .
\end{aligned}
$$

Since $C_{3}$ and $C_{4}$ are independent of $K$ and $\mu>2$, the last inequality gives

$$
\left\|u_{K}\right\|_{L^{\mu}} \leq M_{1} \text {. }
$$

Also, by $(3.14)$ and $\left(H_{4}\right)$ for $W_{K}$, we get

$$
\int_{0}^{T}\left|\nabla W_{K}\left(t, u_{K}\right)\right| d t \leq M_{2}
$$

The inequalities (3.16) and (3.17) yield

$$
\left\|\dot{u}_{K}\right\|_{L^{1}} \leq\left\|B(t) u_{K}\right\|_{L^{1}}+\left\|\nabla W\left(t, u_{K}\right)\right\|_{L^{1}} \leq M_{3} .
$$

Denote $\bar{u}_{K}=\frac{1}{T} \int_{0}^{T} u_{K}(t) d t, \widetilde{u}_{K}=u_{K}-\bar{u}_{K}$. The inequality $\|u\|_{L^{\infty}} \leq c\|\dot{u}\|_{L^{1}}$ (see [11, Proposition 1.1]) for $\widetilde{u}_{K},(3.16)$ and (3.18) provide $K$-independent $L^{\infty}$ bounds for $\bar{u}_{K}$ and $\widetilde{u}_{K}$ respectively. Hence, for some $K_{0}>0,\left\|u_{K}\right\|_{L^{\infty}} \leq K_{0}$. Taking $K>K_{0}$, it follows that $u_{K}$ is a $T$-periodic solution of $(H S)$.

Now, we show that in fact there are infinitely many distinct solutions for $(H S)$. Substituting $k T$ for $T$ in the above, it is easy to see that the problem $(H S)$ possesses a sequence $\left(u_{k}\right)_{k \in \mathbb{N}}$ of $k T$-periodic solutions. Since any $T$-periodic solution is also $k T$-periodic, an additional argument is required to distinguish them. Let $c_{k}=I_{k}\left(u_{k}\right)$, where $I_{k}$ is the functional defined on $E_{k T}$ by

$$
I_{k}(u)=\frac{1}{2} \int_{0}^{k T}(-J \dot{u}-B(t) u, u) d t-\int_{0}^{k T} W_{K}(t, u) d t
$$

where $K$ depends on $k$. Next we prove that $c_{k}$ is bounded from above independently of $k$. Indeed, by (3.13), the corresponding critical point $c_{k}$ satisfies

$$
c_{k} \leq \frac{1}{2} r_{1}^{2}(k),
$$

where $r_{1}(k)$ is determined by the condition

$$
I_{k}(u+r e)=\frac{1}{2}\left(r^{2}-\left\|u^{-}\right\|^{2}\right)-\int_{0}^{k T} W_{K}(t, u+r e) d t \leq 0
$$

for all $r \geq r_{1}(k)$ with $u$ and $e$ adequately chosen. Let $E_{k T}=E_{k}^{+} \oplus E_{k}^{-} \oplus E_{k}^{0}$ be an orthogonal decomposition defined as above for $E_{T}$. Take $u=u^{0}+u^{-} \in E_{k}^{0} \oplus E_{k}^{-}$ and $e \in E_{k}^{+}$be constructed as follows: For $\phi \in E_{1}^{+}=E^{+}$with $\|\phi\|=1$, let

$$
e(t)= \begin{cases}\phi(t), & \text { if } \quad t \in[0, T) \\ 0, & \text { if } \quad t \in[T, k T)\end{cases}
$$


and regard $e$ as $k T$-periodic function defined on $\mathbb{R}$. Then $e \in E_{k}^{+},\|e\|=1$ and particulary $e \not \equiv 0$. Moreover, by $\left(H_{3}\right)$ and (2.3), we have

$$
\begin{aligned}
\int_{0}^{k T} W_{K}(t, u+r e) d t & \geq \int_{0}^{T} W_{K}(t, u+r e) d t \\
& \geq a_{1} \int_{0}^{T}|u(t)+r e(t)|^{\mu} d t-a_{2} T .
\end{aligned}
$$

On the other hand, by Hölder's inequality

$$
\int_{0}^{T}|u(t)+r e(t)|^{2} d t \leq\left[\int_{0}^{T}|u(t)+r e(t)|^{\mu} d t\right]^{2 / \mu}(T)^{\frac{\mu-2}{\mu}} .
$$

Using the orthogonality of $u$ and $e$ in $L^{2}$ and combining (3.21)-(3.23), we get

$$
I_{k}(u+r e) \leq \frac{1}{2}\left(r^{2}-\left\|u^{-}\right\|^{2}\right)-a_{1} T^{\frac{2-\mu}{2}} r^{\mu}\left(\int_{0}^{T}|e(t)|^{2} d t\right)^{\mu / 2}+a_{2} T .
$$

Since $\mu>2$, it follows from (3.24) that there exists $r_{0}$ independent of $k$ such that

$$
I_{k}(u+r e) \leq 0, \quad \text { for all } r \geq r_{0} .
$$

Then $r_{1}(k) \leq r_{0}$ and it follows from (3.20) that

$$
c_{k} \leq \frac{r_{0}^{2}}{2}
$$

Now, if for some $k>m, u_{k}(t) \equiv u(t)=u_{m}(t)$. So $u$ is $k T$-periodic, denote $\frac{k T}{l_{k}}$ its minimal period. By a simple change of variables (see (2.2) in [12]), we obtain

$$
c_{k}=I_{k}\left(u_{k}\right)=l_{k} I_{\frac{k}{l_{k}}}(u) .
$$

Also, $u$ is $m T$-periodic, then we have $\frac{k}{l_{k}}=\frac{m}{l_{m}}$ for some positive integer $l_{m}$ and

$$
c_{m}=I_{m}\left(u_{m}\right)=l_{m} I_{\frac{m}{l_{m}}}(u)
$$

thus

$$
c_{k}=\frac{k}{m} c_{m}
$$

Since $c_{m}>0$ and the sequence $\left(c_{k}\right)$ is bounded, it follows that there can be at most finitely many $k>m$ such that $u_{k}=u_{m}$ for any given $m \in \mathbb{N}$.

Proof of Theorem 1.4. In the proof of Theorem 1.3 the assumption $\left(H_{4}\right)$ is used only in (3.17) to show that $u_{K}$ is bounded in $C^{0}\left(\mathbb{R}, \mathbb{R}^{2 n}\right)$ independently of $K$. Now, by combining (3.14)-(3.16), we obtain the assumption (2.5). Hence, replacing $\left(H_{4}\right)$ by $\left(H_{5}\right)$ in the proof of Theorem 1.3 and using Lemma 2.4, we obtain the same result.

Proof of Theorem 1.7. Let $\mathfrak{L}:=-\left(J \frac{d}{d t}+B\right)$ be the self-adjoint operator acting on $L^{2}\left(S_{k T}, \mathbb{R}\right), k \in \mathbb{N}$, under periodic boundary conditions and $X=D\left(|\mathfrak{L}|^{1 / 2}\right)$ with the norm

$$
\|u\|_{X}=\left|\left\|\left.\mathfrak{L}\right|^{1 / 2} u\right\|_{L^{2}}, \quad \forall u \in X\right.
$$


In [15], it is shown that the condition (1.1) implies $X=E_{k T}$, the norms $\|\cdot\|_{X}$ and $\|\cdot\|_{E_{k T}}$ are equivalents and $X$ has an orthogonal decomposition $X=X^{+} \oplus X^{-}$where the quadratic form $u \longmapsto(\mathfrak{L} u, u)_{L^{2}}$ is positive (resp. negative) definite on $X^{+}$(resp. $X^{-}$). Moreover,

$$
(\mathfrak{L} u, u)_{L^{2}}=\left\|u^{+}\right\|^{2}-\left\|u^{-}\right\|^{2} \text { for all } u=u^{+}+u^{-} \in X=X^{+} \oplus X^{-} .
$$

Recall that $c_{k}=I_{k}\left(u_{k}\right)$ is a critical value of the functional $I_{k}$ defined in (3.19) and $u_{k}$ is the corresponding critical point.

Lemma 3.3. There is a constant $C_{1}>0$ independent of $K$ and $k$ such that

$$
\left\|u_{k}\right\|_{C^{1}} \leq C_{1}
$$

for all $k \in \mathbb{N}$ and $K \geq 1$.

Proof. By (3.25) we know that $c_{k}$ is bounded independently of $K$ and $k$. Moreover, using $\left(H_{6}\right)$ and $\left(H_{1}^{\prime}\right)$, there is a constant $C$ such that

$$
\begin{aligned}
C \geq I_{k}\left(u_{k}\right)= & I_{k}\left(u_{k}\right)-\frac{1}{2} I_{k}{ }^{\prime}\left(u_{k}\right)\left(\widetilde{V} u_{k}\right) \\
= & \int_{0}^{k T}\left(B u_{k}, \widetilde{V} u_{k}-u_{k}\right) d t-\int_{0}^{k T} W_{K}\left(t, u_{k}\right) d t \\
& +\frac{1}{2} \int_{0}^{k T}\left(\nabla W_{K}\left(t, u_{k}\right), \widetilde{V} u_{k}\right) d t \\
\geq & \left(\frac{1}{2}-\frac{1}{\mu}\right) \int_{0}^{k T}\left(\nabla W_{K}\left(t, u_{k}\right), \widetilde{V} u_{k}\right) d t \\
\geq & \left(\frac{\mu}{2}-1\right) \int_{0}^{k T} W_{K}\left(t, u_{k}\right) d t .
\end{aligned}
$$

which implies for certain constant $M>0$

$$
\int_{0}^{k T}\left(\nabla W_{K}\left(t, u_{k}\right), \widetilde{V} u_{k}\right) d t \leq M, \quad \int_{0}^{k T} W_{K}\left(t, u_{k}\right) d t \leq M .
$$

Note that since $W_{K} \geq 0$, by (2.3) and (3.26), we have

$$
\begin{aligned}
M & \geq \int_{0}^{k T} W_{K}\left(t, u_{k}\right) d t \\
& \geq \int_{0}^{T} W_{K}\left(t, u_{k}\right) d t \\
& \geq a_{1} \int_{0}^{T}\left|u_{k}(t)\right|^{\mu} d t-a_{2} T \\
& \geq a_{1} T\left(\min _{t \in S_{T}}\left|u_{k}(t)\right|\right)^{\mu}-a_{2} T \\
& \geq a_{1} T\left(\min _{t \in S_{k T}}\left|u_{k}(t)\right|\right)^{\mu}-a_{2} T .
\end{aligned}
$$

So, inequality (2.6) holds independently of $K$ and $k$ and the proof of Lemma 2.3 goes through with $k T$ replacing $T$. From Lemmas 2.3 and 2.4 , we know 
that $u_{k}$ must be bounded in $C^{0}$ independently of $K$ and $k$. Then, for a sufficiently large $K_{0}$ such that $\left\|u_{k}\right\|_{L^{\infty}}<K_{0}, u_{k}$ is a $C^{1}$ solution of the original problem $(H S)$ and therefore is bounded in $\|\cdot\|_{C^{1}}$.

Lemma 3.4. There is a constant $\delta>0$ independent of $k$ such that

$$
\left\|u_{k}\right\|_{L^{\infty}} \geq \delta \text { for all } k \in \mathbb{N} .
$$

Proof. From the above we may assume that $u_{k}$ is a critical point of the functional defined on $X$ by

$$
J_{k}(u)=\frac{1}{2} \int_{0}^{k T}(-J \dot{u}-B u, u) d t-\int_{0}^{k T} W_{K_{0}}(t, u) d t .
$$

Let $\varepsilon>0$, by the assumption $\left(H_{2}^{\prime}\right)$, there exists $\delta_{\varepsilon}>0$ such that

$$
\left|\nabla W_{K_{0}}(t, x)\right| \leq \varepsilon|x| \quad \text { for }|x| \leq \delta_{\varepsilon} .
$$

Suppose that $\left\|u_{k}\right\|_{L^{\infty}}<\delta_{\varepsilon}$. Writing $u_{k}=u_{k}^{+}+u_{k}^{-} \in X^{+} \oplus X^{-}$and using the definition of $u_{k}$, we obtain

$$
0=J_{k}^{\prime}\left(u_{k}\right)\left(u_{k}^{+}-u_{k}^{-}\right)=\left\|u_{k}\right\|^{2}-\int_{0}^{k T}\left(\nabla W_{K_{0}}\left(t, u_{k}\right), u_{k}^{+}-u_{k}^{-}\right) d t .
$$

Therefore, by (3.26), we get

$$
\begin{aligned}
\left\|u_{k}\right\|^{2} & =\int_{0}^{k T}\left(\nabla W_{K_{0}}\left(t, u_{k}\right), u_{k}^{+}-u_{k}^{-}\right) d t . \\
& \leq \varepsilon \int_{0}^{k T}\left|u_{k} \| u_{k}^{+}-u_{k}^{-}\right| d t \\
& \leq \varepsilon\left\|u_{k}\right\|_{L^{2}}^{2} .
\end{aligned}
$$

By (2.1) and the equivalence of norms, there exists a constant denoted also by $c_{2}$ such that

$$
\left\|u_{k}\right\|^{2} \leq \varepsilon c_{2}\left\|u_{k}\right\|^{2} .
$$

Choosing $\varepsilon<1 / c_{2}$, we get $u_{k}=0$. But this contradicts the fact $J_{k}\left(u_{k}\right)>0$. Then we have $\left\|u_{k}\right\|_{L^{\infty}} \geq \delta_{\varepsilon}$.

Now, we can find a sequence $\left(j_{k}\right)_{k \in \mathbb{N}}$ of integers such that

$$
\max _{t \in[0, T]}\left|u_{k}\left(t+j_{k} T\right)\right|=\max _{t \in \mathbb{R}}\left|u_{k}(t)\right| \in\left[\delta, C_{1}\right] .
$$

Let $\widetilde{u}_{k}(t):=u_{k}\left(t+j_{k} T\right)$. It is easy to see that $\widetilde{u}_{k}$ is a solution of $(H S)$ and $J_{k}\left(\widetilde{u}_{k}\right)=J_{k}\left(u_{k}\right)$. By Lemma 3.3, we can extract a subsequence from any given sequence of integers $k_{n} \longrightarrow \infty$ such that

$$
\widetilde{u}_{k_{n}} \longrightarrow u_{0} \quad \text { as } n \longrightarrow \infty \text { in } \quad C_{l o c}^{1}\left(\mathbb{R}, \mathbb{R}^{2 N}\right),
$$

where $u_{0} \in C^{1}\left(\mathbb{R}, \mathbb{R}^{2 N}\right)$ is a solution of $(H S)$. To conclude the proof of Theorem 1.7, we use the following [15, Lemma 2.9]. 
Lemma 3.5. [15] $u_{0}$ satisfies the following

(a) $u_{0} \neq 0$.

(b) $\quad u_{0} \in L^{p}\left(\mathbb{R}, \mathbb{R}^{2 N}\right)$ for all $p \in[2, \infty]$.

(c) $u_{0} \longrightarrow 0$ as $t \longrightarrow \pm \infty$.

Proof of Theorem 1.8. The proof is based on the following [3, Lemma 1.3].

Lemma 3.6. [3] Suppose $h \in C^{1}\left(\mathbb{R}^{2 N}, \mathbb{R}\right)$ a positive homogenous function of degree $\mu$ with respect to a normalized positive vector field $V$, then for any $x \in \mathbb{R}^{2 N}$,

a) $\nabla h\left(\phi_{s} x\right)=e^{(\mu-2) s} \phi_{s} \nabla h(x)$,

b) $(\nabla h(x), V x)=\mu h(x)$,

c) $\quad h(x) \geq a_{1}|x|^{\mu}$,

d) $|\nabla h(x)| \leq a_{2}|x|^{\mu-1}$,

where $a_{1}, a_{2}$ are positive constants.

Let $W$ a positive homogenous function of degree $\mu>2$ with respect to a normalized positive vector field $V$, then, $W$ satisfies $\left(H_{1}^{\prime}\right)$ via property b) and $\left(H_{2}^{\prime}\right)$ via property d) of Lemma 3.6. Also, by combining properties b), c) and d), it is easy to see that $W$ satisfies $\left(H_{4}\right)$. On the other hand, the assumptions $\left(H_{1}^{\prime}\right)$ and $\left(H_{2}^{\prime}\right)$ imply $\left(H_{1}\right)$ and $\left(H_{2}\right)$ respectively. So, all the hypothesis of Theorem 1.3 are satisfied. Furthermore, if $B$ satisfies the assumptions (1.1) and $\left(H_{6}\right)$, the conclusion of Theorem 1.7 holds.

\section{References}

[1] An, T.: Existence of multiple periodic orbits of Hamiltonian systems on positivetype hypersurfaces in $\mathbb{R}^{2 n}$. J. Math. Anal. Appl. 278, 376-396 (2003)

[2] An, T.: Periodic solutions of superlinear autonomous Hamiltonian systems with prescribed period. J. Math. Anal. Appl. 323, 854-863 (2006)

[3] An, T.: On the minimal periodic solutions of nonconvex superlinear Hamiltonian systems. J. Math. Anal. Appl. 329, 1273-1284 (2007)

[4] Arioli, G., Szulkin, A.: Homoclinic solutions of Hamiltonian systems with symmetry. J. Differ. Equ. 158, 291-313 (1999)

[5] Coti-Zalati, V., Ekeland, I., Séré, E.: A variational approach to homoclinic orbits in Hamiltonian systems. Math. Ann. 288, 133-160 (1990)

[6] Daouas, A., Timoumi, M.: Subharmonics for not uniformly coercive Hamiltonian systems. Nonlinear Anal. 66, 571-581 (2007)

[7] Ding, Y., Girardi, M.: Infnitely many homoclinic orbits of a Hamiltonian system with symmetry. Nonlinear Anal. 38, 391-415 (1999)

[8] Ding, Y., Willem, M.: Homoclinic orbits of a Hamiltonian system. Z. Angew. Math. Phys. 50, 759-778 (1999) 
[9] Hofer, H, Wysocki, K.: First order elliptic system and the existence of homoclinic orbits in Hamiltonian system. Math. Ann. 288, 483-503 (1990)

[10] Long, Y., Zehnder, E.: Morse theory for forced oscillatiotically linear Hamiltonian systems. In: Stochastic Processes, Physics and Geometry, pp. 528-563. World Science Press, Singapore (1990)

[11] Mawhin, J., Willem, M.: Critical point theory and Hamiltonian systems. In: Applied Mathematical Science, vol. 74. Springer, Berlin (1989)

[12] Rabinowitz, P.H.: On subharmonic solutions of Hamiltonian systems. Comm. Pure Appl. Math. 33, 609-633 (1980)

[13] Rabinowitz, P.H.: Minimax methods in critical point theory with applications to differential equations. In: C.B.M.S., vol. 65. AMS, Amsterdam (1986)

[14] Rabinowitz, P.H.: Homoclinic orbits for a class of Hamiltonian systems. Proc. R. Soc. Edinburgh 114(A), 33-38 (1990)

[15] Tanaka, K.: Homoclinic orbits in a first order superquadratic Hamiltonian system: convergence of subharmonic orbits. J. Differ. Equ. 94, 315-339 (1991)

[16] Xu, X.: Periodic solutions for nonautomuous Hamiltonian systems possessing superquadratic potentials. Nonlinear Anal. 51, 941-955 (2002)

[17] Xu, X.: Subharmonics of first order Hamiltonian systems and their asymptotic behaviors. Discrete Continuous Dyn. Syst. Ser. B 3(4), 643-654 (2003)

Adel Daouas

High Institute for Computer Sciences and Telecommunication

Hammam Sousse 4011

Tunisia

e-mail: daouas_adel@yahoo.fr

Present Address:

Department of Mathematics, Faculty of Science

Taibah University

Al-Madinah Al-Munawarah

Kingdom of Saudi Arabia

e-mail: adaouas@taibahu.edu.sa

Received: 21 June 2011.

Accepted: 4 November 2012. 